A section of the same portion of choroid stained with haematoxylin and carbol-fuchsin revealed, after prolonged search, the presence of a bacillus, acid-fast, and with the morphological character of a tubercle bacillus. This conclusion was agreed to by Dr. S. H, Browning who examined the section.

Inoculation Experiment.-Dr. Stanley Griffiths carried out inoculation experiments at the Field Laboratories, Cambridge, from which the following results were obtained :- " Emulsion of mesenteric gland produced tuberculosis in guinea-pig. Cultures of the tubercle bacillus were obtained directly from the mesenteric and bronchial glands. The cultures are dysgonic, i.e., they grow like tubercle bacilli of bovine tuberculosis."

\title{
Conclusion
}

The occurrence of blindness in cats is by no means common, and, as the result of binocular tuberculosis, is distinctly rare. The presence of tuberculosis in both eyes of each of two kittens of the same litter is probably unique. In one case the presence of the tubercle bacillus was demonstrated in the affected choroid of one eye. In the other case, in addition to the presence of tubercle bacillus in the choroid, this organism was found in abundance in a caseous mesenteric lymph gland, and in the spleen; cultures of tubercle bacillus were obtained from bronchial and mesenteric glands; inoculation of a guinea-pig gave a positive tuberculosis result; and lesions of tuberculous nature were found macroscopically in lungs, bronchial glands, spleen, large intestine, and mesenteric gland. The most advanced stage of tuberculosis was found in the mesenteric glands. It is probable that infection took place through the colon. Although no examination of the milk of the dairy of supply was carried out it seems highly probable that the second animal at least was infected from cow's milk.

\section{CASES OF OPHTHALMOLOGICAL INTEREST FROM THE POST-MORTEM RECORDS OF ST. GEORGE'S HOSPITAL, LONDON, I84I-I92I}

BY

\author{
R. R. JAMES \\ LoNDON
}

SoMt few years ago I began to search the post-mortem records of St. George's Hospital to see if I could obtain any facts as to the numbers of those dying in a general hospital of metastatic deposits occurring after removal of an eye affected with malignant disease. 
I was surprised to find so few examples of this condition; no doubt the records of the workhouse infirmaries would be a more fruitful field in which to search, that is, if any are kept in those institutions. I took notes in passing of any findings of ophthalmological interest, and although I failed in my primary object, yet, in view of the excellent way in which the records have been kept, over a long series of years, I thought it might be of some interest to give a brief account of my findings in these memorials of the dead-house.

St. George's is fortunate in possessing an unusually complete series of such records, extending now over a period of more than eighty years, a fact which was strongly emphasized by the late Sir William Osler, who, when he was shown the books, said, that they were by far the best series of post-mortem records with which he was acquainted. The series begins in 1841 , and is complete to date; in 1844, the late Sir Prescott Hewett was appointed curator of the museum and he inaugurated the method of keeping the notes, which method has been followed ever since, and is in use to-day. Hewett devised the plan of writing the post-mortem notes on one side of the page, the left, while on the right-hand side is given a short résumé of the clinical history of the case by the registrar; such a method enables the pathologist to see at a glance the clinical history of any case, and the clinician to see the pathological findings.

During these eighty years nearly 28,000 deaths have been entered on the books and roughly speaking more than two-thirds of this number have undergone a partial or complete post-mortem examination; the eye is not as a rule likely to have been examined if the head was not opened. So far as I am aware, the method of examining the eye has usually been as follows : the roof of the orbit is cut away, the eye defined, and opened at the equator with a pair of sharp-pointed scissors and the posterior half, with a portion of the optic nerve removed and floated out in a dish of water; thus the changes described in this paper are for the most part naked eye changes; very rarely, indeed, is it noted that any microscopical examination has been made; it is obvious that it would be a waste of time embedding and cutting sections of the vast majority of fragments removed in this way.

For my present purpose, it is convenient to divide the material which I have collécted into two sections, cases occurring before 1888 , and those after and including that year. Some curators have been more industrious than others, but, on the whole, a very high average of completeness of examination has been reached.

In the first section it is easiest to give short abstracts of the cases noted; in the second, I propose to group the findings under ophthalmological headings. 
In the first year of the series occurred the case of a male with carcinoma of the antrum and meningitis, and it is noted that the cavernous sinus was full of pus, an example of thrombosis of the cavernous sinus; the specimen is preserved in the museum. Two cases of malignant disease of the antrum, involving the orbit occurred in the years 1845,1846 . In 1847 , an interesting case of tetanus was noted, following a wound of the eye in a male, aged 33 , inflicted by the lash of a whip. The man was admitted early in the year, symptoms of tetanus made their appearance six days after admission, and in four days' time the man was dead. Postmortem, the eye was an abscess, with a perforation of the cornea, and there was infiltration of the orbital tissues. In the same year, in a case of apoplexy, a cyst of the ophthalmic vein, with calcareous wall was found. In 1848, a case of thrombosis of the cavernous sinus following a tonsillar abscess was noted. In 1852, occurred a case of thrombosis of the left optic vein, with protrusion of the eye, secondary to middle ear disease.

In 1853, there was a curious case noted as follows : ptosis, with fixedness of the eyeballs, in a male, aged 36 ; sudden dyspnoea, sphenoid disease. The clinical abstract is as follows: The man was admitted with paralysis of certain of the cranial nerves; dimness of sight had been complained of for six months previous to admission, there was also a history of giddiness and diplopia; ptosis began on the left side and later on was noted on the right side as well ; there was a history of running at the ears some years previously. On admission, the eyeballs were fixed, the axes parallel, the globes motionless; the pupils were noted as acting freely, there was double ptosis, thick speech, defective articulation and some difficulty in swallowing; the loss of power in the fauces increased, and the man died suffocated. The post-mortem notes are as follows : the surface of the body of the sphenoid continuous with the basilar occiput was very injected, it became more porous and more softened on approaching the posterior clinoid processes; two or three small projections of bone existed on the surface just behind the sella Turcica, and the bone forming the posterior surface of the sella was vascular also. The arachnoid and pia were perfectly natural, the dura adherent in places to the calverium; the subarachnoid fluid was slightly increased in amount, and there was an increased amount of fluid in the ven, tricles; the brain, vagus and optic nerves were examined without any abnormality being found ; the eye muscles were noted as being very fatty. This case was reported by the curator, Dr. John Ogle, and will be found in the Transactions of the Pathological Society, vol. 10, p. 224.

The year 1854 saw a great increase in the number of deaths entered on the books from the cholera epidemic which raged that 
year; many of these cases were not examined. In this year occurred a case of a child of three years old with purulent ophthalmia, scarlet fever, and diseased kidneys; the scarlet fever appeared after admission, the kidneys were of the large white variety, and the ophthalmic condition appears to have been phlyctenular conjunctivitis. In the same year, a woman of seventy-three, who died from diffuse inflammation of the forearm is noted as having a cataract.

The year 1857 produced a harvest of cases; a female child of eight years with strumous glands had an old perforated corneal ulcer which had healed with partial staphyloma; a male of thirtyseven with compound fracture of the lower jaw and pyaemia had a hypopyon; a female of twenty-eight had malignant disease of the left frontal bone involving the orbit and displacing the eye; the specimen is in the museum; a male of sixty-five, who had been blind for twelve years, had chronic white softening of the brain and spinal cord; the optic nerves were diminished, pearly in colour, and no healthy nerve tubules could be seen on section; the optic tracts were flattened, thinned, and gelatinous in appearance; the optic thalamus was softened, the corpora quadragemina appeared healthy, and the cerebral vessels were atheromatous.

In 1858, two cases were noted : the first was in a female forty years old, a case of burn; in the right eye the cornea was softened, flaccid and opaque; on turning this structure back, a thick layer of lymph was found on the surface of the iris; the lens was normal. The patient had been in a typhoid state for about three weeks, with occasional bouts of delirium. This appears to me to have been a case of ulcus e lagophthalmo. The second case was that of a child of seven with a malignant tumour of the orbit; he had been admitted in April and he died in October. There was an ulcerating fungating tumour projecting from the left orbit, this tumour was attached to the periosteum at its upper part, but did not involve the bone; the optic nerve was much elongated, and was traced through the tumour to the remains of the eye which lay on the cheek. On dividing the eye, the shrunken choroid was recognized lying loosely enveloped in the sclerotic, which latter was distinct from the growth, though closely adherent to it; the brain and other organs were healthy. The child had been admitted with proptosis and a history of fullness of the upper lid of four months' duration; the growth rapidly increased in size, no operation was performed, and the eye had slowly shrivelled up.

In the next year, in a case of pyaemia, an abscess in the orbit was noted.

In 1861, a female, aged sixty-seven, died of bronchitis after extraction of cataract performed by the late G. D. Pollock; the eye 
was noted as being sound. In the same year, the arcus senilis finds its first mention in a case of injury.

In 1862, a female child of four years was noted as having a fungus haematodes of the eye; evidently this was a case of glioma, secondaries were found in the brain, but the remaining organs were not allowed to be examined.

In 1864, a male, aged forty-four, with pyaemia was noted as having an abscess in the left eyeball; and in the same year, a female, aged twenty-two, with large white kidneys was noted as having opaque whitish retinae; these were not examined microscopically.

In 1867 and 1872 , cases of pyaemia with pus in the eyeball occurred; arcus senilis is mentioned in a middle-aged male; and the case of a lunatic is given, who threw himself out of a window, and who was brought in dead; in this case there was a cicatrix at the inner canthus of the left eye; the eye was shrunken, the choroid showed ossification, and close to the optic disc there was a mass of fibrous tissue which enclosed a small fragment of lead.

In 1873, a case of pyaemia was noted as having serous oedema of the orbital tissues; no pus was found, and the eyes were natural.

In 1874, a male child of twelve years of age, with granular kidneys, showed exudation and haemorrhage in each retina; in the same year in a granular kidney case it was noted that the left eyeball was staphylomatous; the right had been excised at some previous date. Arcus senilis is mentioned in the following year; in 1879 a female twenty-four years of age had purulent meningitis and an abscess in the left orbit; the clinical history of this case had not been written up by the registrar.

In 1880 , a male child thirteen years old had an orbital abscess, with abscess in the frontal lobe and a doubtful fracture of the orbital plate; there was a history of injury four months before.

In 1882, a case of Graves' disease died of emaciation; the prominent globes are mentioned.

In 1883 , occurred a case of anaemia with haemorrhages in the retina; the blood count in this case showed as many white cells as red, the reds were granular.

In 1885 , three cases are noted as having unusually dilated pupils.

In 1888, Dr. F. G. Penrose was appointed curator of the museum; he was a keen clinical observer and used the ophthalmoscope in the wards as a routine; this may account for the sudden increase in the eye findings entered on the post-mortem records.

From this date it is more convenient to arrange the cases in groups.

Injuries.-Excluding ordinary black eyes and conjunctival ecchymosis, the following conditions were found. Laceration of the eye in a case of fractured base. Two cases of ruptured globes 
in fracture of the skull and maxilla respectively. Two cases of bullet wounds of the frontal region, in each of which the eye was collapsed. Six cases of fractured skull in which the orbit is noted as being full of blood, and one case of fractured base in which it is quaintly noted that one of the eyes had been spoilt in former years.

Conjunctiva.-Icteric tint in cases of jaundice is of frequent occurrence. Chemosis is noted thrice. A case of streptococcal conjunctivitis occurred in a baby a month old who died of gastroenteritis. Purulent ophthalmia is noted in a case of erysipelas of the head.

Cornea.-Bilateralulcers were noted in an infant dead of bronchopneumonia. Opacity of the cornea was noted in the case of a young female dead of diabetes. Vicious ulcers were noted in a baby, aged eight months, with acute nephritis. Cornea decomposed in a case of generalized tuberculosis. Nebula in a case of morbus cordis. Corneal opacities with anterior staphyloma in a baby dead of pneumonia. Opaque corneae, shrunken globes and blepharitis in a baby who was dead from broncho-pneumonia. Arcus senilis was found in many cases.

Iris.--Old iritis was noted in a case of rupture of the bladder. Irido-cyclitis occurred once in a case of pericarditis and septicaemia.

Lens.-Cataract is only mentioned four times.

Choroid.-Tubercle occurs for the first time in 1888; the case was one of phthisis in a male, aged thirty-one; besides this, there are twenty-three cases of choroidal tubercle in cases of generalized tuberculosis. It is interesting to note that in a few cases of this condition, three or four at most, the eyes are expressly stated to have shown no signs of choroidal tubercle. Two cases of choroiditis were noted, one in a male dead of gastric carcinoma, and the other in a case of morbus cordis, in which much atrophy of the choroid was noted as well. Neoplasm. Benign. One case of melanoma of the choroid which was diagnosed during life by myself in a female who was admitted unconscious, and who died of apoplexy. Malignant. All were melanotic. A male, aged thirty-seven, who died of secondaries in 1896, the eye having been removed for tumour at the Royal Eye Hospital in 1894; a male of thirty-one, who died of deposits in the liver in 1899, the eye having been removed at the Royal London Ophthalmic Hospital in 1897. A male, aged sixty-four, died of secondaries in the liver in 1919 with the primary growth in the eye still in situ; he had had some treatment at another hospital some months earlier, but the eye had not been removed, nor had the growth burst through the sclerotic. This is a suitable spot in which to place the case of a male of forty-five who died of malignant disease 
of the dura mater, and of the cervical glands in 1899, whose eye had been removed for hypopyon at St. George's in the previous year; the eye does not appear to have been examined after removal, at least I could find no record of its having been done.

Retina.-Albuminuric retinitis was noted in thirty-three cases. Retinal haemorrhage in cases of renal disease not indexed as albuminuric retinitis was found in seventeen cases. Retinal haemorrhage in cases of anaemia was noted in the following cases : aplastic, one case; pernicious, five cases; idiopathic anaemia, three cases; leucocythaemia, two cases; purpura haemorrhagica, three cases; haemorrhagic diathesis, one case. Retinitis pigmentosa was noted in one case. Opaque fibres in the retina were found in one case. Retinal haemorrhages were noted as being found in the following cases: pneumonia, meningitis, malignant disease of the sternum, emphysema with fatty heart, two cases of surgical kidney, fractured skull, cystitis with fatty heart, cerebral haemorrhage, spinal meningitis with arrested sexual development, calculus obstruction of the ureters, diabetes, sarcoma of the base of the skull, choroidal sarcoma, toxaemia, and in eight cases of morbus cordis. One case of small white kidney was noted as having healthy retinae. Detachment of the retina occurred in one case only, it was in a male, aged twenty, who died of small white kidney and heart failure, the retinal condition had been diagnosed during life; this retina was examined microscopically, and found to be full of exudation and haemorrhage.

Orbit.-Abscess was found in five cases; malignant disease noted in seven cases, for the most part either in cases of apoplexy or of fractured skull. Distension of the sheath was found occasionally in cases of meningitis and of cerebral tumour. The optic nerve was torn across in one case of fractured skull. Optic neuritis was found in sixteen cases of cerebral tumour, in four cases of cerebral abscess, in four cases of apoplexy, with disease of the cerebral arteries and sinuses, in five cases of chronic renal disease, in six cases of meningitis, and in two cases of pyaemia. Optic atrophy, apart from atrophy of the nerve in cases where the eye had been previously removed was noted in six cases.

Orbit.-Abscess was found in five cases; malignant disease in one case; exophthalmos was noted in two cases of Graves' disease; phthisis bulbi was noted in two cases, and panophthalmitis in one case of cerebro-spinal fever.

Both eyes were noted as destroyed in a baby of three, dead of broncho-pneumonia, and the eyes were noted as glistening in a case of prussic acid poisoning.

I am afraid that little of real importance emerges from this research into the post-mortem records; it is interesting to note the extraordinarily varied number of morbid conditions in which 
retinal haemorrhage wasfound. No one can be attached to a general hospital for very long without quickly becoming cognizant of this fact, and it is one which might with advantage be more emphasized than it is in our textbooks. The case of tetanus is interesting. Of course it occurred many years ago, and although nothing is said either in the case records or in the post-mortem notes of there being any other wound to account for it than the wound of the eye, it is possible, in fact I should think it very probable, that some other focus of infection existed. There can be no doubt of the tetanus, our forefathers in the profession knew tetanus when they saw it, but I do not think that I can count this case definitely among the very few which have been recorded where the wound in the eye was the primary cause.

The prominence of the globes noted in all three cases of Graves' disease shows that those who maintain that the exophthalmos does not persist in these cases after death are incorrect.

I have to express my best thanks to Dr. Robert Donaldson, Curator of the museum and Pathologist to the Hospital, for permission to extract these notes from the records, and for much kindly help.

\title{
A SERIES OF ONE HUNDRED CASES OF CATARACT REMOVED UNDER A SUBCONJUNCTIVAL BRIDGE
}

\author{
BY THE LATÉ \\ Charles Killick, M.D., F.R.C.S. \\ BRADFORD
}

No one, I think, should have the temerity to lay down the law about the merits or drawbacks of any particular operation unless he has performed it a great number of times. As I have, in the course of a fairly long professional career, extracted some 1,500 cataracts, $\mathrm{I}$ am in a position to know something about my subject and may claim to be acquainted with its various difficulties and complications. There can hardly be one that I have not come up against, I must have committed every possible fault and perpetrated every blunder known to the ophthalmic surgeon, including inserting the knife with its cutting edge downwards on no less than three occasions, until at last I have entered upon the calm waters of efficiency I trust, so far at any rate as cataract extraction is concerned. - My practice has always been to make the incision with puncture and counter-puncture at the limbus, to include nearly 